\title{
Autocorrelation-Invariant Discrete-time Functions and Associated Orthogonal Sequences
}

Authors: P. Bréhonnet, N. Tanguy, P. Vilbé, L-C. Calvez

LEST-UMR CNRS 6165,

Université de Bretagne Occidentale,

6 av. Le Gorgeu C.S. 93837,

29238 Brest Cedex 3,

France

Tel: +33298017157

Fax: +33298016395

Corresponding author: Pascale Bréhonnet

E-mail: Pascale.Brehonnet@univ-brest.fr

Abstract: The paper studies frequency domain characterization and properties of autocorrelation discrete time functions. Such functions may be useful in the design and synthesis of signaling waveforms with prescribed autocorrelation functions.

Keywords: Autocorrelation; Orthogonal functions; Discrete-time functions 


\section{Introduction}

It has long been known that the orthonormal set of causal discrete time functions known as the discrete Laguerre functions [3,4] can be generated by passing $l_{0}[n] \bumpeq\left(1-a^{2}\right) a^{n} U[n]$, a decaying causal exponential ( $U[n]$ denotes the unit step function), through a cascade of $i$ identical all-pass discrete-time filters having transfer function

$T(z)=\frac{1-a z}{z-a},|a|<1$

to generate the $i^{\text {th }}$ member of the set. Then each member has the same autocorrelation function since they have identical energy spectra. Furthermore, this autocorrelation $\phi[k]$ is identical to $l_{0}[k]$ for $k \geq 0$. That is, $\phi[k]=l_{0}[k \mid]$.

Thus $l_{0}[n]$ is a function which is, in this sense, invariant under the operation of autocorrelation. For this reason we call $l_{0}[n]$ an Autocorrelation-Invariant Discrete (AID) time function. A similar curiosity which holds for continuous-time Laguerre functions has led Gonsalves [2] to investigate an associated orthogonal set of Autocorrelation-Invariant Continuous time functions. AI functions find their use in the design and synthesis of signaling waveforms. For example, if certain specifications on the autocorrelation function of a discrete-time signal can be met by an AID function $f_{0}[k]$ for $k \geq 0$, then $f_{0}[n]$ is immediately an acceptable discrete-time waveform and so are the other members of the orthogonal set derived from $f_{0}[n]$ which have the same autocorrelation function. Orthonormal functions have been used for reducing the complexity of an echo canceler [6]. Some applications of Laguerre functions can be found in the references given in [7].

The purpose of this paper is to investigate the existence and properties of a class $C_{A I D}$ of real causal finite energy discrete-time functions $f[n]$ with rational z-transform $F(z)$ and autocorrelation function

$\phi_{f f}[k]=f[k \mid]$.

\section{Frequency domain characterization}

Considering the z-transform of (1) yields

$$
F(z) F\left(\frac{1}{z}\right)=F(z)+F\left(\frac{1}{z}\right)-f[0] \text {. }
$$

Without loss of generality, the rational z-transform $F(z)$ can be written as

$$
F(z)=1-R(z)
$$

where $R(z)$ is an irreducible rational function

$$
R(z) \hat{=} \frac{P(z)}{A(z)}
$$

with $\operatorname{deg}(P) \leq \operatorname{deg}(A)$. On account of the finite energy assumption, $R(z)$ has all its poles inside the unit circle in the z-plane. Substituting (3) into (2) yields

$$
R(z) R\left(\frac{1}{z}\right)=1-f[0]
$$


Assuming $f[0]=1$ implies $R(z)=0$ and then $F(z)=1$, a trivial solution of (2) which presents no particular interest. Therefore, we will subsequently assume $f[0] \neq 1$ which implies $\operatorname{deg}(P)=\operatorname{deg}(A)$, because in case $\operatorname{deg}(P)<\operatorname{deg}(A)$, the initial value theorem for the $\mathrm{z}$ transform applied to (3) yields $f[0]=1$.

In view of (4), (5) may be written

$$
P(z) P\left(\frac{1}{z}\right)=(1-f[0]) A(z) A\left(\frac{1}{z}\right) \text {. }
$$

Multiplying both sides by $z^{m}, m=\operatorname{deg}(A)=\operatorname{deg}(P)$, and denoting by $A^{R}(z) \hat{=} z^{m} A(1 / z)$ the reciprocal polynomial, we obtain

$$
P(z) P^{R}(z)=(1-f[0]) A(z) A^{R}(z)
$$

Because $P(z)$ and $A(z)$ are relatively prime $(R(z)$ is irreducible by assumption), for a given stable polynomial $A(z)$ with non-zero constant term the only acceptable solution is

$$
P(z)=\lambda A^{R}(z)
$$

where $\lambda$ denotes a real constant to be determined. Notice that if the constant term in $A(z)$ is zero, there is no $P(z)$ for which (6) holds. Therefore, in the following, we will assume that the constant term in the polynomial $A(z)$ is non-zero. Substituting (7) into (6) yields $\lambda^{2}=1-f[0]$ where $f[0]$ depends on $\lambda$. Indeed, in view of (3) and (4)

$F(z)=1-\lambda \frac{A^{R}(z)}{A(z)}$.

Applying the initial value theorem for the z-transform of a causal function yields

$f[0]=1-\lambda \alpha_{m}$

with

$\alpha_{m}=\lim _{z \rightarrow \infty} \frac{A^{R}(z)}{A(z)}=\frac{A(0)}{A^{R}(0)}$

hence

$\lambda^{2}=\lambda \alpha_{m}$

and the only acceptable solution is $\lambda=\alpha_{m}$. Therefore

$F(z)=1-\alpha_{m} \frac{A^{R}(z)}{A(z)}$.

With the polynomial $A_{m-1}(z)$ defined as

$$
z A_{m-1}(z) \hat{=} A(z)-\alpha_{m} A^{R}(z)
$$

(9) can be rewritten as

$$
F(z)=\frac{z A_{m-1}(z)}{A(z)}
$$

It is worth noting that $A_{m-1}(z)$ is the first polynomial generated when testing stability of $A(z)$ with the widely used Aström-Raible table [1,5]. Thus, every stable polynomial $A(z)$ with non-zero constant term readily yields a function $f \in C_{A I D}$ for which (1) holds. This function is given by its z-transform (10). 
An orthogonal set $\left\{f_{i}[n]\right\}$ can be generated by passing $f[n] \hat{=} f_{0}[n]$ through identical all-pass filters in Laguerre fashion, each filter having transfer function $T(z)=A^{R}(z) / A(z)$. Some of the interesting properties of these functions are summarized in the following section.

\section{AID-time functions properties}

In the following, the cross-correlation function of two real finite-energy discrete-time functions $f_{i}[n]$ and $f_{j}[n]$, denoted by $\phi_{i j}[k]$, is defined as

$\phi_{i j}[k] \hat{=} \sum_{n=-\infty}^{+\infty} f_{i}[n] f_{j}[n+k]$

If the real causal finite-energy discrete-time functions $f_{i}[n]$ have the z-transforms $F_{i}(z)=\frac{z A_{m-1}(z)}{A(z)}\left[\frac{A^{R}(z)}{A(z)}\right]^{i}, i=0,1,2, \ldots$

where $A(z)$ is a stable polynomial of degree $m \geq 1$ with non-zero constant term, $A^{R}(z)$ is the reciprocal polynomial and $A_{m-1}(z)$ is the first polynomial, of degree $m-1$, generated via Aström-Raible table, then the following properties are verified:

$1^{\circ}$ ) The autocorrelation function of $f_{0}[n]$ is

$\phi_{00}[k]=f_{0}[|k|]$

hence $f_{0} \in C_{\text {AID }}$. This has been proved in section 2 .

Because an autocorrelation function is maximum at the origin, the following holds

$\mid f_{0}[|k|] \leq f_{0}[0]=1-\alpha_{m}^{2}$

with $\alpha_{m}$ defined by (8).

$2^{\circ}$ ) Each member of the set $\left\{f_{i}[n]\right\}$ has the same autocorrelation function

$\phi_{i i}[k]=f_{0}[|k|]$

On account of $A^{R}(1 / z) / A(1 / z)=A(z) / A^{R}(z)$ the following holds for the energy spectra

$\Phi_{i i}(z)=F_{i}(z) F_{i}\left(\frac{1}{z}\right)=F_{0}(z) F_{0}\left(\frac{1}{z}\right)$

which in turn yields

$\phi_{i i}[k]=\phi_{00}[k]$.

As a corollary notice that all the $f_{i}[n]$ have the same energy

$\left\|f_{i}\right\|^{2}=\sum_{n=0}^{\infty}\left|f_{i}[n]\right|^{2}=\phi_{i i}[0]=f_{0}[0]=1-\alpha_{m}{ }^{2}$.

Figures 1 and 2 illustrate the auto and cross-correlation for a set of three functions in the case where $f_{0}$ is a decaying exponential $(A(z)=z-0.5)$. The theoretical infinite sequences have been truncated to 20 terms. 

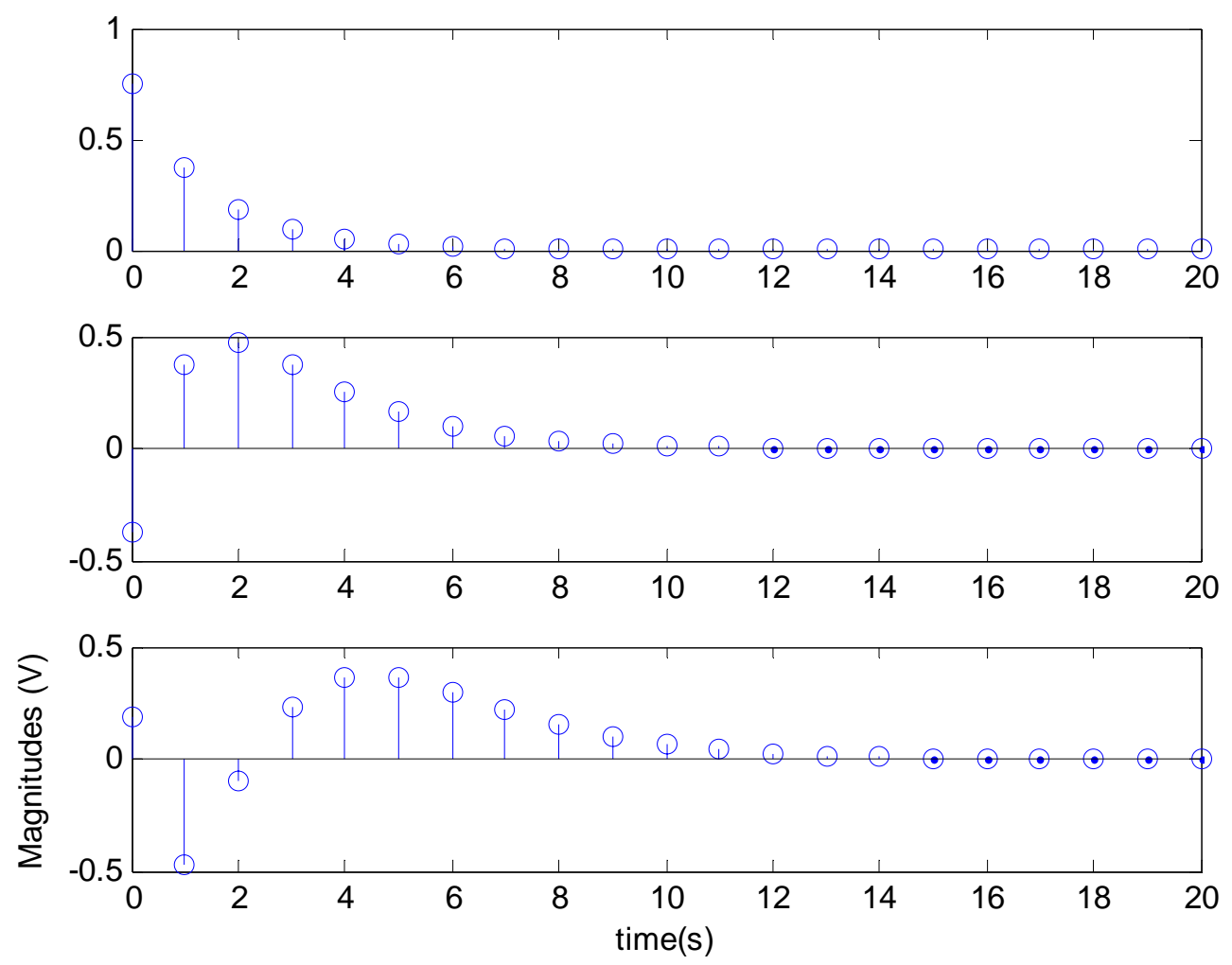

Figure 1 - Time functions $f_{0}, f_{1}, f_{2}$

$3^{\circ}$ ) The cross correlation function of $f_{i}[n]$ and $f_{j}[n]$ is $\phi_{i j}[k]=f_{j-i}[k]-\alpha_{m} f_{j-i-1}[k], j>i$

which depends only on the difference $j-i$ and is zero for $k \leq 0$.

This can be demonstrated as follows. The cross-energy spectrum of $f_{i}[n]$ and $f_{j}[n]$ is $\Phi_{i j}(z)=F_{i}\left(\frac{1}{z}\right) F_{j}(z)$

$$
\begin{aligned}
& =\left(1-\alpha_{m} \frac{A(z)}{A^{R}(z)}\right)\left(1-\alpha_{m} \frac{A^{R}(z)}{A(z)}\right)\left(\frac{A^{R}(z)}{A(z)}\right)^{j-i} \\
& =\left(1-\alpha_{m} \frac{A^{R}(z)}{A(z)}\right)\left[\left(\frac{A^{R}(z)}{A(z)}\right)^{j-i}-\alpha_{m}\left(\frac{A^{R}(z)}{A(z)}\right)^{j-i-1}\right] \\
& =F_{j-i}(z)-\alpha_{m} F_{j-i-1}(z)
\end{aligned}
$$

and hence, after z-transform inversion, the above expression of the cross-correlation function results.

Because $\phi_{i j}[k]$ is a linear combination of two causal functions, it equals zero for $k<0$. Letting $k=0$

$\phi_{i j}[0]=f_{j-i}[0]-\alpha_{m} f_{j-i-1}[0], j>i$

The initial value theorem for the z-transform leads to $f_{i}[0]=\lim _{z \rightarrow \infty} F_{i}(z)=\left(1-\alpha_{m}{ }^{2}\right) \alpha_{m}{ }^{i}$ 
from which $\phi_{i j}[0]=0$ follows (for $j>i$ ).

Corollary: the $\left\{f_{i}[n]\right\}$ form an orthogonal set.

The following orthogonality relation holds $\sum_{n=0}^{+\infty} f_{i}[n] f_{j}[n+k]=0, i>j, k \geq 0$.

The infinite sum on the left hand side is $\phi_{i j}[k]=\phi_{j i}[-k]$ which in turn equals zero since $-k \leq 0$ and $i>j$ (see at the beginning of this $3^{\circ}$ ). When $k=0$ the above relation is valid for both cases $j>i$ and $j<i$ because interchanging $i$ and $j$ is then possible. When $i=j$ the sum reduces to $\phi_{i i}[0]=f_{0}[0]=1-\alpha_{m}{ }^{2}$ and finally

$\sum_{n=0}^{+\infty} f_{i}[n] f_{j}[n]=\left(1-\alpha_{m}^{2}\right) \delta_{i j}$

where $\delta_{i j}$ is the Krönecker symbol ; thus the $\left\{f_{i}[n]\right\}$ form an orthogonal set.
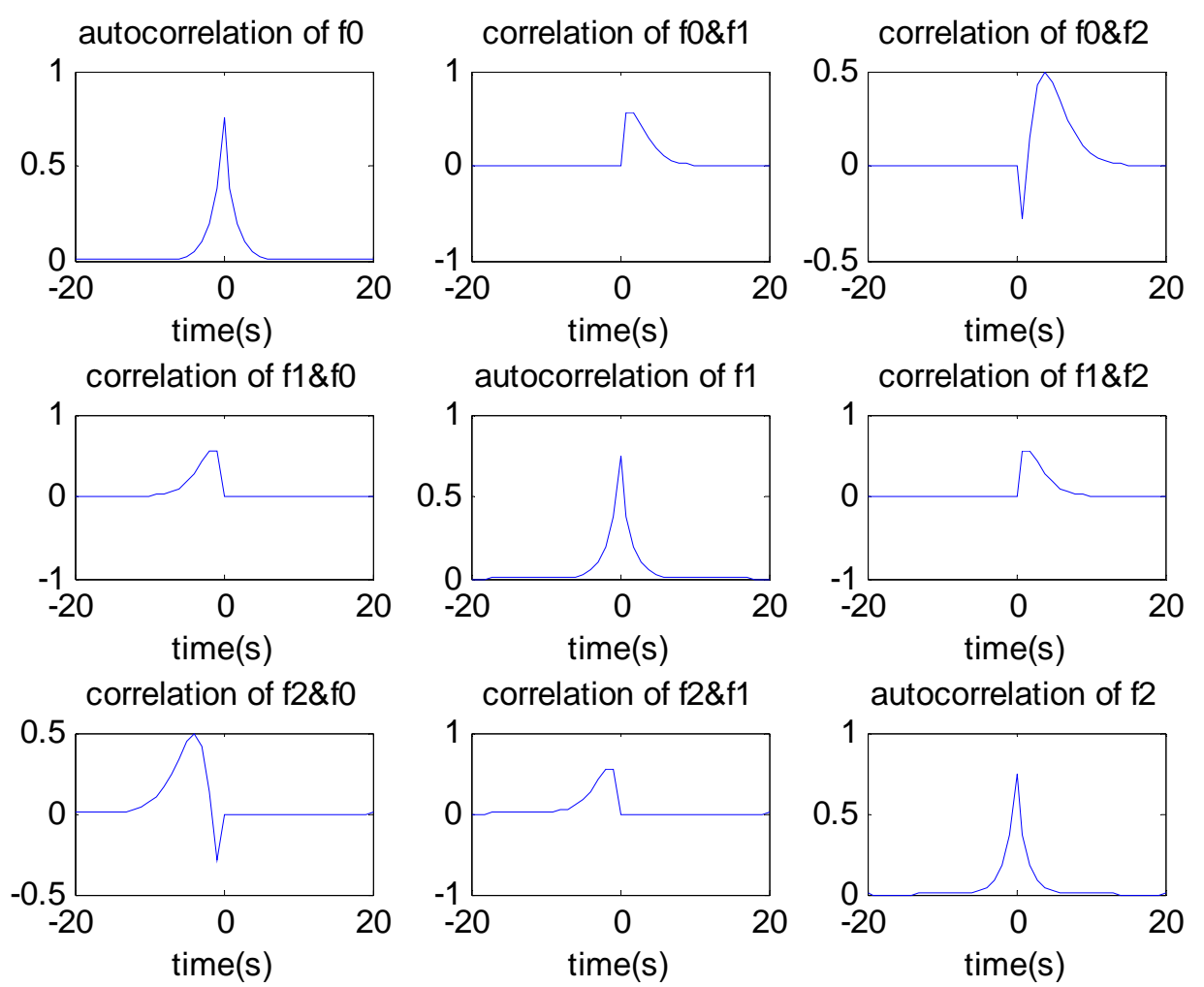

Figure 2 - auto and cross-correlation functions of $f_{0}, f_{1}, f_{2}$

We can see on figure 2 that all functions $f_{i}[n]$ for $i=0,1,2$ have the same autocorrelation. The cross-correlation of $f_{i}[n]=f_{0}[n]$ and $f_{j}[n]=f_{1}[n]$ on one hand and that of $f_{i}[n]=f_{1}[n]$ and $f_{j}[n]=f_{2}[n]$ on the other hand are the same. This is due to property 3 , cross-correlation only depends on the difference $j-i=1$.

$\phi_{i j}[k]=f_{j-i}[k]-\alpha_{m} f_{j-i-1}[k], j>i$

which depends only on the difference $j-i$ and is zero for $k \leq 0$.

$4^{\circ}$ ) If $f_{0}[n]$ has a zero at $n=N$, then $f_{i}[n]$ and $f_{i}[n+N]$ are orthogonal. 
Indeed, from $2^{\circ}$

$\sum_{n=0}^{+\infty} f_{i}[n] f_{i}[n+k]=f_{0}[k], k>0$

Letting $k=N$, orthogonality of $f_{i}[n]$ and $f_{i}[n+N]$ follows from $f_{0}[N]=0$.

Corollary: the zero crossings of $f_{0} \in C_{A I D}$ cannot be periodic

The proof is by contradiction. The integers $N_{1}, N_{2}, \ldots, N_{q}, \ldots$ are zero crossings of $f_{0}[n]$ if for all $q \geq 1$

a) $f_{0}\left[N_{q}\right]=0$ and $f_{0}\left[N_{q}-1\right] f_{0}\left[N_{q}+1\right]<0$

b) $f_{0}[n]$ has constant sign in the interval $\left[0, N_{1}\right.$ [ and in any of the intervals $] N_{q}, N_{q+1}[$. Assume that the zero crossings are given by the infinite sequence $N, 2 N, 3 N, \ldots$ Hence, for any $n \neq m N, \quad m=0,1,2, \ldots$, the product $f_{0}[n] f_{0}[n+N]$ is strictly negative which implies $\sum_{n=0}^{+\infty} f_{0}[n] f_{0}[n+N]<0$ : a contradiction follows by $\sum_{n=0}^{+\infty} f_{0}[n] f_{0}[n+N]=f_{0}[N]=0$ which achieves the proof.

$\left.5^{\circ}\right)$ The discrete convolution of $f_{i}[n]$ with $f_{j}[n]$ is $f_{i}[n] * f_{j}[n]=f_{i+j}[n]-\alpha_{m} f_{i+j+1}[n]$ which depends only on the sum $i+j$.

Starting with the z-transform $F_{i}(z) F_{j}(z)$, the proof is similar to that of the cross-correlation property in $3^{\circ}$.

$6^{\circ}$ ) Consider a second function $g_{0} \in C_{A I D}$ and let $g_{j}[n]$ have the z-transform

$G_{j}(z)=\left[1-\beta_{r} \frac{B^{R}(z)}{B(z)}\right]\left[\frac{B^{R}(z)}{B(z)}\right]^{j}$

with $B(z)$ a stable polynomial of degree $r \geq 1$ with non-zero constant term, $\beta_{r}=B(0) / B^{R}(0)$, and let $c[n]$ denote the discrete convolution $c[n]=c_{i j}[n]=f_{i}[n] * g_{j}[n]$.

Then, the autocorrelation of $c[n]$ is given by the nice relationship

$\phi_{c c}[k]=f_{0}[|k|] * g_{0}[|k|]$

where $f_{0}[|k|]$ and $g_{0}[|k|]$ are respectively recognized as the autocorrelation functions of $f_{i}[n]$ and $g_{j}[n]$.

This can be demonstrated as follows. Because $C(z)=F_{i}(z) G_{j}(z)$ the energy spectrum $\Phi_{c c}(z)=C(z) C(1 / z)$ may be rearranged as

$$
\begin{aligned}
\Phi_{c c}(z) & =F_{i}(z) F_{i}(1 / z) G_{j}(z) G_{j}(1 / z) \\
& =\left[F_{0}(z) F_{0}(1 / z)\right]\left[G_{0}(z) G_{0}(1 / z)\right] \\
& =Z\left\{f_{0}\lfloor|n|]\right\} Z\left\{g_{0}[|n|]\right\}
\end{aligned}
$$

Z-transform inversion leads to the expression (11) and the proof is achieved. The property can be easily generalized for more than two functions.

Since the autocorrelation function of $c_{i j}[n]$ is independent of $i, j$, all the $c_{i j}[n]$ have the same energy i.e. 
$\left\|f_{i} * g_{j}\right\|^{2}=\left\|f_{0} * g_{0}\right\|^{2}$

$7^{\circ}$ ) No two functions in $C_{A I D}$ can be orthogonal.

Letting $k=0$ in the relationship concerning convolution

$$
\begin{aligned}
\phi_{c C}[0] & =\sum_{n=-\infty}^{\infty} f_{0}[|n|] g_{0}[|0-n|] \\
& =2\left(\sum_{n=0}^{\infty} f_{0}[n] g_{0}[n]\right)-f_{0}[0] g_{0}[0]
\end{aligned}
$$

but, $f_{0}[0]=\left\|f_{0}\right\|^{2}$ and similarly $g_{0}[0]=\left\|g_{0}\right\|^{2}$; therefore

$$
\begin{aligned}
\sum_{n=0}^{\infty} f_{0}[n] g_{0}[n] & =\frac{1}{2}\left(\phi_{c c}[0]+\left\|f_{0}\right\|^{2}\left\|g_{0}\right\|^{2}\right) \\
& =\frac{1}{2}\left(\left\|f_{0} * g_{0}\right\|^{2}+\left\|f_{0}\right\|^{2}\left\|g_{0}\right\|^{2}\right)
\end{aligned}
$$

and the inner product of two arbitrary functions $f_{0}$ and $g_{0}$ in $C_{A I D}$ is strictly positive : two functions in $C_{A I D}$ cannot be orthogonal..

\section{Conclusion}

In this paper, the existence and interesting properties of Autocorrelation-Invariant Discrete (AID) time functions with prescribed spectrum have been established. Associated sets of orthogonal sequences with identical spectrum are readily derived. The derived properties may find their use in the design and implementation of communication systems.

\section{References}

[1] K.J. Aström, Introduction to stochastic control theory, Academic Press, Mathematics in Science and Engineering, vol. 70, chap. 5 (1970) 128-138.

[2] R.A. Gonsalves, Autocorrelation-Invariant Functions and an Associated Orthogonal Set of Functions, Proc. of the IEEE 52 (6) (1964) 737-738.

[3] M.J. Gottlieb, Concerning some polynomials orthogonal on a finite or enumerable set of points, American Journal of Mathematics 60 (2) (1938) 453-458.

[4] R.E. King, P.N. Paraskevopoulos, Digital Laguerre filters, International Journal of Circuit Theory and Applications 5 (1977) 81-91.

[5] M.R. Raible, A simplification of Jury's tabular form, IEEE Trans. Autom. Control 19 (3) (1974) 248-250.

[6] G.W. Davidson, D.D. Falconer, Reduced Complexity Echo Cancellation Using Orthonormal Functions, IEEE Trans. On Circuits and Systems, vol 38 (1), (1991) 21-28

[7] N. Tanguy, R. Morvan. P. Vilbé, and L.C. Calvez, Online Optimization of the Time Scale in Adaptative Laguerre-Based Filters, IEEE Trans. On Signal Processing, vol 48 (4), (2000) 1184-1187. 